\title{
Die Trennung des Tellurs vom Antimon.
}

\author{
Von \\ W. MUthuana und E. SChröDer.
}

(Mitteilung aus dem Chemischen Laboratorium der kgl. Akademie der Wissenschaften in München.)

Das Antimon ist bekanntlich ein häufiger Begleiter des Tellurs; die wichtigeren ungarischen Tellurerze, also namentlich Schrifterz und Blättererz (Nagyágit) sind antimonhaltig; und bei der Analyse dieser interessanten Mineralien hat man demnach eine Trennung der beiden Elemente auszuführen. Bis jetzt kennt man nur zwei $\mathrm{Me}-$ thoden, um diesen Zweck zu erreichen; sie leiden indessen beide an gewissen Mängeln und sind schwierig auszuführen. Die erste beruht auf der Fällbarkeit des Tellurs in salzsaurer Lösung durch Schwefeldioxyd; sie ist die am häufigsten benutzte, aber durchaus nicht einwandfrei, wie es auf den ersten Blick scheinen könnte. Es ist längst bekannt, dals man Tellur als Präparat durch Ausfällen mit Schwefeldioxyd nicht rein erhalten kann; enthält nämlich eine Lösung Tellur und Antimon nebeneinander, so wird ein Teil des letzteren immer mit gefällt werden, selbst wenn die Menge der freien Salzsäure eine sehr grolse (bis $20 \%$ ) ist. Schon aus diesem Grunde ist die Methode für quantitative Zwecke zu verwerfen. RosE ${ }^{1}$ schlägt vor, die Lösung mit Weinsäure zu versetzen, indessen haben wir uns überzeugt, dafs auch diese Methode das Mitfallen des Antimons nicht verhindert. Wir verfuhren in der Weise, dafs wir reines $\mathrm{TeO}_{2}$ und $\mathrm{Sb}_{2} \mathrm{O}_{3}$ in etwa $20 \%$ iger Salzsäure lösten und dann nach Zusatz von etwa $1 \mathrm{~g}$ Weinsäure auf $0.1 \mathrm{~g}$ der Mischung in der Siedehitze Schwefeldioxyd einleiteten; der schwarze, flockige Niederschlag enthielt ziemlich bedeutende Mengen Antimon.

1 Analytische Chemie (6. Anfl.), 2. Teil, S. 438. 
Eine zweite Methode wäre die von WöHLER, ${ }^{1}$ welcher die feingepulverten Tellurerze mit verkohltem Weinstein unter Luftabschlufs einige Stunden im Tiegel glüht, mit Wasser auszieht und das in Lösung gegangene Tellurkalium durch Einblasen von Luft zersetzt; es wird sodann das Tellur in metallischem Zustand abgeschieden. Dies Verfahren kann wegen der leichten Zersetzbarkeit des Telluralkalis keine guten Resultate geben. Wir haben (zur Trennung von Tellur und Selen nach der Cyankaliummethode) öfter Tellurkaliumlösungen unter den Händen gehabt, und beobachtet, dafs man eine solche Lösung nicht von einem Gefäls ins andere giefsen kann, ohne dafs eine Abscheidung von Tellur stattfindet. Es dürfte also sebr schwierig, wenn nicht unmöglich sein, die WöHLER'sche Schmelze zu extrahieren und den kohlehaltigen Rückstand mit Wasser auszuwaschen, obne dals Tellur sich abscheidet und in Verlust gerät.

Unsere Methode, welche wir bei der Analyse von Blättererzen mit dem besten Erfolge anwendeten, berubt auf der leichten Zersetzlichkeit des Schwefeltellurs und der Unlöslichkeit des entstehenden Tellurs in mälsig konz. Salzsäure. Ein Tellursulfid dürfte überhaupt in reinem Zustand nicht zu erhalten sein, wir verweisen in dieser Beziehung auf die Beobachtungen von BECKER, ${ }^{2}$ die wir in allen Einzelheiten bestätigen können. Leitet man in eine Tellurdioxyd enthaltende salzsaure Lösung Schwefelwasserstoff ein, so bewirkt jede Blase zuerst eine rotbraune Trübung, die nach wenigen Sekunden in eine schwarze Fällung sich verwandelt; die entstandene schwarze Substanz ist nichts als ein mechanisches Gemenge von Tellur und Schwefel. Das Tellur ist nun in einer Salzsäure, deren Gehalt an $\mathrm{HCl} 20 \%$ nicht übersteigt, völlig unlöslich, während sich Antimonsulfid leicht auflöst, und schien uns diese Verschiedenheit im Verhalten der beiden Körper ein gutes Mittel zur Trennung, was sich auch als richtig herausstellte. Zunächst wurde die Methode an Mischungen von $\mathrm{Sb}_{2} \mathrm{O}_{3}$ und $\mathrm{TeO}_{2}$ erprobt, und dabei in folgender Weise verfahren: Die mit Weinsäure versetzte salzsaure Lösung der beiden Oxyde wird durch mehrstündiges Einleiten von Schwefelwasserstoff völlig gefällt, und der ausgewaschene Niederschlag mit Schwefelkaliumlösung in der Wärme digeriert; es geht dann alles in Lösung, das Tellur als Sulfotellurit. Diese Lösung, die nicht zuviel überschüssiges Schwefelkalium enthalten darf, wird nun unter Um-

1 Mineralanalyse in Beispielen (2. Aufl.), S. 109.

2 Lieb. Ann. (1876) 180, 257. 
rühren in heifse, $20 \%$ ige Salzsäure, die ebenfalls Weinsäure enthält, eingegossen, und einige Zeit digeriert, bis der Niederschlag flockig geworden ist und sich völlig abgesetzt hat. Man filtriert sodann ab, wäscht zunächst mit heifser Salzsäure, sodann mit weinsäurehaltigem Wasser, bis das Filtrat kein Antimon mehr enthält, und bestimmt die so getrennten Metalle nach den gewöhnlichen Methoden. Den Tellur und Schwefel enthaltenden Niederschlag mufs man in rauchender Salpetersäure lösen und das Tellur als Metall oder als Dioxyd wägen.

Die Analysen gaben folgende Resultate:

\begin{tabular}{|c|c|c|c|c|}
\hline & $\begin{array}{c}\text { Angewandte } \\
\text { Substanz }\end{array}$ & Gefunden & Differenz & $\begin{array}{l}\text { Differenz auf } \\
\text { die Oxyde } \\
\text { berechnet }\end{array}$ \\
\hline & & $\left\{\begin{array}{l}0.1007 \mathrm{~g} \mathrm{Te}= \\
0.1261 \mathrm{~g} \mathrm{TeO}_{2}\end{array}\right\}$ & $0.0021 \mathrm{~g}$ & $-0.61 \%$ \\
\hline 1. & $0.1316 \mathrm{~g} \mathrm{Sb_{2 } \mathrm { O } _ { 3 }}$ & $\left\{\begin{array}{l}0.1528 \mathrm{~g} \quad \mathrm{Sb}_{2} \mathrm{~S}_{\mathrm{g}}= \\
\left.0.13095 \mathrm{~g} \mathrm{Sb_{2 } \mathrm { O } _ { 3 }}\right\}\end{array}\right\}$ & $0.00065 \mathrm{~g}$ & $-0.25 \%$ \\
\hline & $0.0927 \mathrm{~g} \mathrm{TeO}_{2}$ & $\left\{\begin{array}{l}0.0736 \mathrm{~g} \mathrm{Te}= \\
0.09215 \mathrm{~g} \mathrm{TeO}_{2}\end{array}\right\}$ & $0.00055 \mathrm{~g}$ & $-0.36 \%$ \\
\hline 11. & $0.0595 \mathrm{~g} \mathrm{Sb_{2 }} \mathrm{O}_{3}$ & $\left\{\begin{array}{l}0.0688 \mathrm{~g} \quad \mathrm{Sb}_{2} \mathrm{~S}_{3}= \\
0.05899 \mathrm{~g} \quad \mathrm{Sb}_{2} \mathrm{O}_{3}\end{array}\right\}$ & $0.00051 \mathrm{~g}$ & $-0.33 \%$ \\
\hline III & $0.1526 \mathrm{~g} \mathrm{TeO}$ & $\left\{\begin{array}{l}0.1212 \mathrm{~g} \mathrm{Te}= \\
0.15175 \mathrm{~g} \mathrm{TeO}\end{array}\right\}$ & $0.00085 \mathrm{~g}$ & $-0.31 \%$ \\
\hline & $0.1233 \mathrm{~g} \mathrm{Sb_{2 }} \mathrm{O}_{3}$ & $\left\{\begin{array}{l}0.1437 \mathrm{~g} \quad \mathrm{Sb}_{2} \mathrm{~S}_{3}= \\
\left.0.12317 \mathrm{~g} \mathrm{Sb_{2 } \mathrm { O } _ { 3 }}\right\}\end{array}\right\}$ & $0.00013 \mathrm{~g}$ & $-0.05 \%$ \\
\hline
\end{tabular}

Die Resultate sind also verhältnismälsig recht befriedigende und jedenfalls bessere, als sie mit einer der anderen Methoden erhalten werden können; man mufs indessen genau nach obiger Vorschrift verfahren und darf besonders nicht versäumen, den beim Eingiefsen der Schwefelkaliumlösung in Salzsäure erhaltenen Niederschlag auch auf dem Filter noch zweimal mit heilser Salzsäure zu waschen, da sich dem Tellur sonst unfehlbar Antimon beimengen würde.

\section{Analyse eines Blättererzes von Nagyág.}

Das von uns untersuchte Mineral verdanken wir der Liebenswürdigkeit von Herrn Prof. Dr. P. Grotu; dasselbe stammt von einer sehr reichen, in der hiesigen mineralogischen Sammlung des Staates befindlichen Stufe. Es war schön krystallisiert, soweit man 
sehen konnte völlig einheitlich und lag in Form. von dünnen, biegsamen, sehr weichen, bleigrauen Blättchen vor; die qualitative Analyse ergab $\mathrm{Pb}, \mathrm{Au}, \mathrm{Sb}, \mathrm{Te}, \mathrm{S}$; keine Spuren von $\mathrm{Ag}$, $\mathrm{Cu}$ und $\mathrm{Fe}$; wir hatten also sehr reines Material zur Verfügung. Wir haben zwei Analysen ausgeführt; bei der ersten wurde das Mineral mit rauchender Salpetersäure im Einschmelzrohr bei $260^{\circ}$ aufgelöst, das zweite Mal einfach mit Königswasser auf dem Wasserbade behandelt. Aus der salzsauren, mit Weinsäure versetzten Lösung wurde zunächst mit Oxalsäure das Gold gefällt, aus dem Filtrat mit Schwefelwasserstoff Blei, Antimon und Tellur abgeschieden, der Niederschlag mit Schwefelkalium digeriert, und schliefslich $\mathrm{Sb}$ und $\mathrm{Te}$ wie oben angegeben getrennt. Den Schwefel haben wir jedesmal in einer besonderen Portion bestimmt.

Die Analysen gaben folgende Resultate:

I.

Angewendet $0.4286 \mathrm{~g}$.

$\mathrm{Pb}$

$\mathrm{Au}$

$\mathrm{Sb}$

Te

$\mathrm{S}$

Gangart (Quarz)
$53.55 \%$

9.47 ,

6.05,

18.99 "

11.895,

$\frac{0.56 \%}{100.515 \%}$
II.

Angewendet $0.5539 \mathrm{~g}$.

$53.76 \%$

9.56

- , 1

19.51,

12.40,

-

Die etwas höheren Zahlen bei Analyse II sind dem Umstande zuzuschreiben, dafs keine Gangart vorhanden war.

Bringt man bei Analyse I den Quarz in Abrechnung und dividiert die Prozente durch die Atomgewichte, so erhält man:

$$
\begin{aligned}
& \mathrm{Pb}: \mathrm{Au}: \mathrm{Sb}: \mathrm{Te}: \\
&=0.260: 0.0483: 0.0507: 0.1504: 0.3825 \\
&=10.4: 1.932: 2.028: 6.015: 15.3 .
\end{aligned}
$$

Auffallend ist bei diesem Verhältnis, dafs sich $\mathrm{Au}: \mathrm{Sb}: \mathrm{Te}$ ziemlich genau wie 2:2:6 verhalten; man könnte also die Formel

$$
\mathrm{Sb}_{2} \mathrm{Te}_{3} \cdot \mathrm{Au}_{2} \mathrm{Te}_{3} .5 \mathrm{~Pb}_{2} \mathrm{~S}_{3}
$$

aufstellen. Die Annahme eines Bleisesquisulfides, das dem $\mathrm{Pb}_{2} \mathrm{O}_{3}$ entsprechen würde, erscheint allerdings befremdend, da eine solche Verbindung bis jetzt nicht bekannt ist. Will man davon absehen, so könnte man unter der Voraussetzung, dals etwas zuviel Schwefel gefunden wurde, folgende Formel aufstellen:

$$
8 \mathrm{PbS} .2 \mathrm{PbTe} .2 \mathrm{AuTe}_{2} . \mathrm{Sb}_{2} \mathrm{~S}_{5} \text {. }
$$

\footnotetext{
${ }^{1}$ Konnte infolge eines Unfalles leider nicht gewogen werden.
} 
Die in der Natur vorkommenden Sulfide, Arsenide, Telluride u. s. w. der Schwermetalle besitzen häufig eine Zusammensetzung, welche sich mit der Valenztheorie nicht in Einklang bringen lälst. Wir erinnern nur an den Schwiefelkies $\mathrm{FeS}_{2}$, den Arsenkies FeAsS, den Kobaltglanz CoAsS; ferner den Melonit, der nach GENTH der Hauptsache nach aus $\mathrm{Ni}_{2} \mathrm{Te}_{3}$ besteht; im Schrifterz mufs man die ganz abnormen Verbindungen $\mathrm{AgTe}_{2}$ und $\mathrm{AuTe}_{2}$ annehmen; es lielsen sich diese Beispiele leicht vermehren. Wir halten es daher im vorliegenden Falle für zwecklos, die obigen Formeln für das Blättererz näher zu diskutieren und begnügen uns mit der empirischen $\mathrm{Zu}$ sammensetzung $\mathrm{Pb}_{10} \mathrm{Au}_{2} \mathrm{Sb}_{2} \mathrm{Te}_{6} \mathrm{~S}_{15}$.

Jedenfalls dürfte diese Formel der wahren Zusammensetzung des Minerales sehr nahe kommen, da wir mit ganz einwurfsfreiem Material gearbeitet baben und die Analysen mit grörster Sorgfalt ausgeführt wurden. Die in letzter Zeit veröffentlichten Untersuchungen von SIPöcz ${ }^{1}$ und $\mathrm{H}_{A N K O}{ }^{2}$ wurden mit eisenhaltigem, also wohl nicht vollkommen einheitlichem Material ausgeführt; auch stand den Genannten die oben beschriebene gute Trennungsmethode nicht zur Verfügung.

1 Tschermak's Mineral. und petrogr. Mitt. (1886) 7, 267.

2 Zeitschr. Krystallogr. 17, 514.

Bei der Redaktion eingegangen am 23. Januar 1897. 